\title{
Impact of Low Molecular Weight Heparin on Overall Survival in Patients with Advanced Lung Cancer: A Retrospective Study
}

\author{
Rammey Hassan $^{1}$, Shuchi Gulati ${ }^{1}$, Yellu Mahender ${ }^{1}$, Ihab Eldessouki ${ }^{1}$, Nabeela Iffat Siddiqi ${ }^{1}$, \\ Changchun Xie ${ }^{2}$, Jane Pruemer ${ }^{3}$, Nagla Abdel Karim ${ }^{1}$ \\ ${ }^{1}$ Department of Oncology, University of Cincinnati Medical Center, Cincinnati, USA \\ ${ }^{2}$ Division of Biostatistics and Bioinformatics, University of Cincinnati, Cincinnati, USA \\ ${ }^{3}$ James L. Winkle College of Pharmacy, University of Cincinnati, Cincinnati, USA
}

Email address:

karimnf@ucmail.uc.edu (N. A. Karim)

\section{To cite this article:}

Rammey Hassan, Shuchi Gulati, Yellu Mahender, Ihab Eldessouki, Nabeela Iffat Siddiqi, Changchun Xie, Jane Pruemer, Nagla Abdel Karim. Impact of Low Molecular Weight Heparin on Overall Survival in Patients with Advanced Lung Cancer: A Retrospective Study. American Journal of Clinical and Experimental Medicine. Vol. 5, No. 5, 2017, pp. 173-175. doi: 10.11648/j.ajcem.20170505.13

Received: July 20, 2017; Accepted: August 14, 2017; Published: September 4, 2017

\begin{abstract}
Low Molecular Weight Heparin (LMWH) is approved for treatment of and secondary prophylaxis against thromboembolic events in patients with cancer. However, its role in improving Overall Survival (OS) remains unclear. A retrospective study to evaluate effect of LMWH on OS in advanced lung cancer was conducted by retrospectively identifying patients with advanced lung cancer (stage IIIA, IIIB and IV) including non-small cell, small cell and rare histopathologic subtypes from 2004 to 2014. Patients with early stage lung cancer and those with incomplete information were excluded. Data on patient demographics (age, gender, ethnicity), histopathology, staging, medications (including chemotherapy and anticoagulants) and patient outcome were collected. Death was considered as the final endpoint. Patients with and without LMWH use were identified. This study did not find an improvement in OS with use of LMWH in advanced stage lung cancer patients. Even though the results were not statistically significant, there was a trend towards higher mortality in patients treated with LMWH for thrombosis compared to patients without thrombosis. Future prospective studies using a larger patient population should evaluate the impact of prophylactic versus therapeutic LMWH on OS in advanced lung cancer.
\end{abstract}

Keywords: Lung Cancer, Low Molecular Weight Heparin, Venous Thromboembolism, Thrombosis

\section{Introduction}

Lung cancer is the leading cause of cancer deaths among both males and females in America [1]. It is also the leading cause of cancer death worldwide, and has surpassed breast cancer as the leading cause of cancer death among females in more developed countries [2]. Since the time of Trousseau it has been well established that cancer puts an individual in a hypercoagulable state, thus increasing the risk for thrombotic complications. As in any cancer, venous thromboembolism (VTE) is common in lung cancer patients and may be precipitated by venous stasis, activation of clotting cascades by pro-coagulant tumor factors, and by vascular injury due to chemotherapy and central venous catheters [3]. Subcutaneous low molecular weight heparin (LMWH) is approved for and has replaced intravenous unfractionated heparin in the treatment of acute VTE. The long-term use of LMWH is recommended to prevent the recurrence of VTE [3]. Despite advances in the understanding of malignancy and thromboembolic events, there are still limitations in clinical management being that patients (cancer and non-cancer) still die from vascular thromboembolic events even when diagnosed and appropriately managed [4]. Venous thromboembolism (VTE) incidence is around 40-100 cases per 1000 person-years in lung carcinoma patients in contrast to $1-2$ cases per 1000 person-years in the general population [5]. However, one of the challenges with vascular thromboembolism is that true prevalence is underestimated due to many cases being clinically unapparent [4]. The 
prevalence of VTE in cancer patients has been estimated as up to $15 \%$ antemortem, and higher postmortem owing to the asymptomatic nature of many episodes of VTE [6]. This poses a problem as VTE itself is not only a common coexisting entity but a direct cause of death in cancer patients, in fact it is the second leading cause of death in patients with cancer [7].

\section{Hypothesis}

We hypothesized that the use of anticoagulation as LMWH improves the Overall Survival (OS) in patients with advanced lung cancer.

\section{Aims and Method}

The objective of this study was to assess the overall survival (OS) of patients with advanced lung cancer receiving LMWH. In a single academic-based institution via an electronic medical record we retrospectively identified patients with advanced lung cancer (stage IIIA, IIIB and IV) including non-small cell, small cell and rare histopathologic subtypes from 2004 to 2014. Patients with early stage lung cancer and those with incomplete information were excluded. Data was collected on patient demographics (age, gender, ethnicity), histopathology, staging, medications (including chemotherapy and anticoagulants) and patient outcome. Death was considered as the final endpoint. Patients with and without LMWH use were identified.

\section{Statistical Analysis}

Kaplan-Meier method was used to calculate median OS and $95 \%$ CI. Cox model was used to test treatment effect adjusted for age and gender. A subgroup analysis was subsequently performed for patients on LMWH with history of thrombosis. Data was analyzed using $\mathrm{SAS}^{\circledR}$ software Ver 9.4 (SAS Institute Inc., Cary, NC, USA).

\section{Results}

Of the 197 patients with advanced lung cancer that were reviewed, 28 received LMWH. Their mean age at diagnosis was $62.2 \pm 10.8$ years. The median OS of patients who received LMWH was 15 months (460 days) (95\% CI: 157, 1051) and that of the non LMWH group was 17.7 months (539 days) (95\% CI: 376, 916), hazard ratio (HR) 1.335 (p= 0.339).

The patients with advanced lung cancer who needed the LMWH due to thrombotic event $(\mathrm{N}=19)$ had a lower OS 10.5782 months (322 days) $(95 \% \mathrm{CI}: 157,1051)$ compared to the patients who received LMWH at any point of their disease without the presence of a thrombotic event. The Maximum Likelihood Estimate for this subgroup after controlling for covariates showed a HR $1.202(p=0.751)$. None of the LMWH patients had a major bleeding event as a cause of death.

\section{Discussion}

The association of cancer with VTE has long been identified and studied. Between 1970 and 1982, 23,796 standardized autopsies were performed in an urban Swedish population to investigate the presence of VTE in patients with various types of cancer. The relationship between cancer and PE was evaluated with logistic regression showing overall PE prevalence was $23 \%$, and $10 \%$ of the population had a fatal PE [8]. Till this day there are still fatal pulmonary embolisms in cancer patients. The importance of understanding this association will not only aid in improving overall survival but quality of life as well. Though the role of LMWH on OS in patients with advanced malignancy and known VTE has not been demonstrated thoroughly, many studies show the overall benefit of LMWH in patients with advanced malignancy without known VTE.

On review of the literature, Kakkar et al. showed for patients with advanced malignancy who had a better prognosis, alive 17 months after randomization, KaplanMeier survival estimates at 2 and 3 years from randomization were significantly improved for patients receiving dalteparin versus placebo (78\% v 55\% and $60 \%$ v 36\%, respectively, $P$ $=.03$ ). Although dalteparin administration did not significantly improve 1-year survival rates, they suggest the observed improved survival in the subgroup of patients with a better prognosis shows a potential modifying effect of dalteparin on tumor biology [9]. This study showed higher OS in patients treated with LMWH without thrombosis compared to the subgroup of patients treated with LMWH for thrombosis.

Klerk et al. evaluated the effect of low molecular weight heparin on survival in patients with advanced malignancy without venous thromboembolism by randomly assigning patients to receive a 6 week course of subcutaneous nadroparin or placebo. In the a priori specified subgroup of patients with a life expectancy of 6 months or more at enrollment, the hazard ratio was 0.64 (95\% CI, 0.45 to 0.90$)$ with a median survival of 15.4 and 9.4 months, respectively [10]. Again, suggesting LMWH favorably influences survival in patients with advanced malignancy.

Altinbas et al. sought to determine whether addition of low-molecular-weight heparin (LMWH) to combination chemotherapy (CT) would improve SCLC outcome compared with $\mathrm{CT}$ alone. Overall tumor response rates were $42.5 \%$ with CT alone and $69.2 \%$ with CT plus LMWH $(P=$ $0.07)$. Median progression-free survival was 6.0 months with CT alone and 10.0 months with CT plus LMWH $(P=0.01)$. Median overall survival was 8.0 months with $\mathrm{CT}$ alone and 13.0 months with CT plus LMWH $(P=0.01)$ [11].

Lebeau et al. randomized 277 patients with SCLC to either receive or not to receive subcutaneous heparin injections for 5 weeks in addition to one of two chemotherapy regimens. In comparison to the 139 patients who did not receive heparin, the 138 patients who received anticoagulant treatment obtained better complete response rates $(37 \%$ vs. $23 \%, \mathrm{P}=$ 0.004 ), better median survival (317 days vs. 261 days, $\mathrm{P}=$ 
0.01 ), and better survival rates at 1,2 , and 3 years (40\% vs. $30 \%, 11 \%$ vs. $9 \%, 9 \%$ vs. $6 \%$, respectively) [12].

\section{Conclusion}

Most of the reviewed literature aimed to determine the benefit of LMWH on overall survival in patients with advanced malignancy. This study is unique in that it sought out to determine if LMWH had a benefit to OS for patients with advanced lung malignancy with thrombosis compared to without thrombosis. The other studies did not make this distinction, however, but showed the benefit of LMWH on patients with advanced malignancy even suggesting that it may modify tumor biology. Perhaps with more research, the addition of LMWH to a treatment regimen can be considered.

This study did not find an improvement in OS with use of LMWH in advanced stage lung cancer patients. Even though the results were not statistically significant, there was a trend towards higher mortality in patients treated with LMWH for thrombosis compared to patients without thrombosis. Our findings further demonstrate the need for future prospective studies using a larger patient population to evaluate the impact of prophylactic versus therapeutic use of anticoagulation as LMWH on OS in advanced lung cancer.

\section{References}

[1] Siegel RL, Miller KD, Jemal A. Cancer statistics, 2016. CA Cancer J Clin. 2016; 66(1): 7-30. doi: 10.3322/caac.21332.

[2] Torre LA, Bray F, Siegel RL, Ferlay J, Lortet-Tieulent J, Jemal A. Global cancer statistics, 2012. CA Cancer J Clin. 2015; 65(2): 87-108. doi: 10.3322/caac.21262 [doi].

[3] Bréchot JM. Thrombosis and Lung Cancer, 2005; 22(6 Pt 2): 8S33-7. PMID: 16340833
[4] Maraveyas A, Johnson M. Does clinical method mask significant VTE-related mortality and morbidity in malignant disease? $B r \quad J$ Cancer. 2009; 100(12): 1837-1841. doi: 10.1038/sj.bjc.6605091.

[5] Tesselaar ME, Osanto S. Risk of venous thromboembolism in lung cancer. Curr Opin Pulm Med. 2007; 13(5): 362-367. doi: 10.1097/MCP.0b013e328209413c.

[6] Johnson MJ, Sproule MW, Paul J. The prevalence and associated variables of deep venous thrombosis in patients with advanced cancer. Clin Oncol (R Coll Radiol). 1999; 11(2): 105-110.

http://www.ncbi.nlm.nih.gov/pubmed/10378636.

[7] Khorana AA. Venous thromboembolism and prognosis in cancer. Thromb Res. 2010; 125(6): 490-493. doi: 10.1016/j.thromres.2009.12.023.

[8] Ogren M, Bergqvist D, Wåhlander K, Eriksson H, Sternby NH. Trousseau's syndrome - What is the evidence? A population-based autopsy study. Thromb Haemost. 2006; 95(3): 541-545. doi: 10.1160/TH05-10-0694.

[9] Kakkar AK, Levine MN, Kadziola Z, et al. Low molecular weight heparin, therapy with dalteparin, and survival in advanced cancer: The fragmin advanced malignancy outcome study (FAMOUS). J Clin Oncol. 2004; 22(10): 1944-1948. doi: 10.1200/JCO.2004.10.002.

[10] Klerk CP, Smorenburg SM, Otten HM, et al. The effect of low molecular weight heparin on survival in patients with advanced malignancy. J Clin Oncol. 2005; 23(10): 2130-2135. doi: 10.1200/JCO.2005.03.134.

[11] Altinbas M, Coskun HS, Er O, et al. A randomized clinical trial of combination chemotherapy with and without lowmolecular-weight heparin in small cell lung cancer. $J$ Thromb Haemost. 2004; 2(8): 1266-1271. doi: 10.1111/j.15387836.2004.00871.x.

[12] Lebeau B, Chastang C, Brechot JM, et al. Subcutaneous heparin treatment increases survival in small cell lung cancer. "Petites Cellules" Group. Cancer. 1994; 74(1): 38-45. 